\title{
Elastic scattering of twisted electrons by atomic target: Going beyond the Born approximation
}

\author{
V. P. Kosheleva, ${ }^{1}$ V. A. Zaytsev, ${ }^{1}$ A. Surzhykov, ${ }^{2,3}$ V. M. Shabaev, ${ }^{1}$ and Th. Stöhlker ${ }^{4,5,6}$ \\ ${ }^{1}$ Department of Physics, St. Petersburg State University, \\ Universitetskaya naberezhnaya 7/9, 199034 St. Petersburg, Russia \\ ${ }^{2}$ Physikalisch-Technische Bundesanstalt, D-38116 Braunschweig, Germany \\ ${ }^{3}$ Technische Universität Braunschweig, D-38106 Braunschweig, Germany \\ ${ }^{4}$ GSI Helmholtzzentrum für Schwerionenforschung GmbH, D-64291 Darmstadt, Germany \\ ${ }^{5}$ Helmholtz-Institute Jena, D-07743 Jena, Germany \\ ${ }^{6}$ Institut für Optik und Quantenelektronik, \\ Friedrich-Schiller-Universität, D-07r43 Jena, Germany
}

\begin{abstract}
The elastic scattering of twisted electrons by neutral atoms is studied within the fully relativistic framework. The electron-atom interaction is taken into account in all orders, thus allowing us to explore high-order effects beyond the first Born approximation. In order to illustrate these effects, detailed calculations of the total and differential cross sections as well as the degree of polarization of scattered electrons are performed. Together with the analysis of the effects beyond the first Born approximation, we discuss the influence of the kinematic parameters of the incident twisted electrons on the angular and polarization properties of the scattered electrons.
\end{abstract}

PACS numbers: 03.65.Pm, 34.80.Lx 


\section{INTRODUCTION}

The twisted (or vortex) electron beams being predicted [1] and realized [2 4] less than a decade ago presently attract a lot of attention from both experimental and theoretical sides (for a review, see Ref. [5]). In contrast to the conventional (plane-wave) electrons, the twisted electrons possess a non-zero projection of the orbital angular momentum (OAM) $\hbar m$ onto their propagation direction. This projection, which nowadays can be as high as $1000 \hbar$ [6], determines the magnitude of the OAM induced magnetic moment. Due to such huge (as opposite to the plane-wave electrons) magnetic dipole moment the twisted electrons are presently regarded as a valuable tool for studying magnetic properties of materials at nano scale [7-10]. Moreover, with the growth of $m$ the effects induced by the complex interplay between the spin and OAM become more pronounced. All these have stimulated the studies on the interaction of vortex electrons with ionic and atomic targets [11-15].

In the present paper, we focus on one of fundamental scattering processes, namely, the elastic (Mott) scattering of twisted electrons by neutral atoms. This process can be used to better understand the effects induced by a complex interplay between the spin and orbital magnetic moments. These effects can be studied, as an example, by means of measuring magnetic circular dichroism in the elastic and inelastic scattering of the vortex electron beams [7, 10, 16, 17], where the spin-orbit interaction plays a crucial role. The case of the Mott scattering by heavy atoms is of particular interest. Indeed, for such systems the spin-orbit interaction is greatly enhanced. Moreover, the Mott scattering is presently regarded as a tool for diagnostics of vortex electrons [18], thus stimulating an additional interest to the elastic scattering of twisted electrons.

The Mott scattering of twisted electrons by atoms has been studied in Ref. [14] within the first Born approximation. This approximation is well justified for light atomic systems and relatively large velocities of the incident electron. In the case of the elastic scattering of the twisted electrons by heavy atoms, the description which accounts for the electron-atom interaction in all orders is demanded. We present such description in this paper. Our approach is based on the nonperturbative treatment of the electron-atom interaction discussed in Ref. [15]. In the framework of this method we evaluate the differential and total cross sections for the Mott scattering of the twisted electrons at various atomic targets. The degree of longitudinal polarization for the process considered is also calculated. Additionally, we compare our results with predictions obtained within the first Born approximation [14]. It is found that even for light $Z$ systems reliable results 
for the total cross section of the Mott scattering of the twisted electrons with energies around (and lower) $100 \mathrm{keV}$ can be obtained only within the all-order relativistic treatment.

The paper is organized as follows. In Sec. IIA the basic equations for the Mott scattering of the plane-wave electrons are briefly recalled. In Sec. IIB the theoretical description of the Mott scattering of the twisted electrons accounting for the electron-atom interaction in all orders is presented. The comparison of the results obtained within the first Born approximation with the all-order relativistic ones is presented in Secs. IIIA and IIIB. Effects of kinematic properties of the incident twisted electron are considered in Sec. IIIC. Finally, a summary and an outlook are given in Sec. IV.

Relativistic units, $m_{e}=\hbar=c=1$, and the Heaviside charge unit, $e^{2}=4 \pi \alpha$ ( $\alpha$ is the fine structure constant), are used in the paper.

\section{BASIC FORMALISM}

The theory of the elastic scattering of the plane-wave electrons by ionic and atomic targets is well established and presented in many textbooks (see, e.g., Ref. [19]). The elastic scattering of the twisted electrons has been considered so far only in the framework of the first Born approximation in Ref. [14]. The approach used in that work can not be utilized for the calculations beyond this approximation. Here we describe the formalism which allows one to study the Mott scattering of the twisted electrons without perturbation expansion in terms of the coupling constant describing the electron-atom interaction. This formalism is similar to one appearing in the plane-wave case. Let us start, therefore, with the description of the elastic scattering of the plane-wave electrons.

\section{A. Mott scattering of the conventional (plane-wave) electrons}

The differential cross section (DCS) for the elastic scattering of an electron with the asymptotic four-momentum $\left(\varepsilon, \mathbf{p}_{i}\right)$ and the helicity $\mu_{i}$ (spin projection onto its momentum direction) can be expressed as

$$
\frac{d \sigma_{\mu_{f} \mu_{i}}^{(\mathrm{pl})}}{d \Omega}=\left|\tau_{\mu_{f} \mu_{i}}^{(\mathrm{pl})}\right|^{2}
$$


where $\mu_{f}$ is the helicity of the scattered electron possessing four-momentum $\left(\varepsilon, \mathbf{p}_{f}\right)$. The amplitude $\tau_{\mu_{f} \mu_{i}}^{(\mathrm{pl})}$ of the scattering process can be written as follows [20]

$$
\tau_{\mu_{f} \mu_{i}}^{(\mathrm{pl})}=\sqrt{(2 \pi)^{3}} U_{p_{f} \mu_{f}}^{\dagger}(\hat{\mathbf{n}}) G_{\mu_{i}}^{(+)}(\hat{\boldsymbol{\nu}}, \hat{\mathbf{n}}) .
$$

Here $\hat{\boldsymbol{\nu}}=\mathbf{p}_{i} /\left|\mathbf{p}_{i}\right|, \hat{\mathbf{n}}=\mathbf{p}_{f} /\left|\mathbf{p}_{f}\right|, G_{\mu_{i}}^{(+)}(\hat{\boldsymbol{\nu}}, \hat{\mathbf{n}})$ is the bispinor amplitude whose explicit form will be specified below, and $U_{p_{f} \mu_{f}}(\hat{\mathbf{n}})$ is the Dirac bispinor [21]:

$$
U_{p_{f} \mu_{f}}(\hat{\mathbf{n}})=\frac{1}{\sqrt{2 \varepsilon}}\left(\begin{array}{l}
\sqrt{\varepsilon+1} \chi_{1 / 2 \mu_{f}}(\hat{\mathbf{n}}) \\
\sqrt{\varepsilon-1}(\boldsymbol{\sigma} \cdot \hat{\mathbf{n}}) \chi_{1 / 2 \mu_{f}}(\hat{\mathbf{n}})
\end{array}\right)
$$

where $\boldsymbol{\sigma}$ is the vector of Pauli matrices, $\chi_{1 / 2 \mu_{f}}(\hat{\mathbf{n}})$ satisfies $(\mathbf{S} \cdot \hat{\mathbf{n}}) \chi_{1 / 2 \mu_{f}}(\hat{\mathbf{n}})=\mu_{f} \chi_{1 / 2 \mu_{f}}(\hat{\mathbf{n}})$ with $\mathbf{S}=\boldsymbol{\sigma} / 2$ being the spin operator.

The bispinor amplitude $G_{\mu_{i}}^{(+)}(\hat{\boldsymbol{\nu}}, \hat{\mathbf{n}})$ can be obtained from the asymptotic behavior of the incident electron wave function

$$
\Psi_{\mathbf{p}_{i} \mu_{i}}^{(+)}(\mathbf{r}) \underset{r \rightarrow \infty}{\longrightarrow} \psi_{\mathbf{p}_{i} \mu_{i}}(\mathbf{r})+G_{\mu_{i}}^{(+)}(\hat{\boldsymbol{\nu}}, \hat{\mathbf{r}}) \frac{e^{i p r}}{r}
$$

where $p \equiv\left|\mathbf{p}_{i}\right|=\left|\mathbf{p}_{f}\right|, \hat{\mathbf{r}}$ is the unit vector in the $\mathbf{r}$ direction, and $\psi_{\mathbf{p}_{i} \mu_{i}}(\mathbf{r})$ is the plane-wave solution of the free Dirac equation:

$$
\psi_{\mathbf{p}_{i} \mu_{i}}(\mathbf{r})=\frac{e^{i \mathbf{p}_{i} \cdot \mathbf{r}}}{\sqrt{(2 \pi)^{3}}} U_{\mathbf{p}_{i} \mu_{i}}(\hat{\boldsymbol{\nu}})
$$

The wave function $\Psi_{\mathbf{p}_{i} \mu_{i}}^{(+)}(\mathbf{r})$ describing the scattering of the electron in the central potential is given by $[22$ 24]:

$$
\Psi_{\mathbf{p}_{i} \mu_{i}}^{(+)}(\mathbf{r})=\frac{1}{\sqrt{4 \pi \varepsilon p}} \sum_{\kappa m_{j}} C_{l 01 / 2 \mu_{i}}^{j \mu_{i}} i^{l} \sqrt{2 l+1} e^{i \delta_{j, l}} D_{m_{j} \mu_{i}}^{j}\left(\varphi_{\hat{\boldsymbol{\nu}}}, \theta_{\hat{\boldsymbol{\nu}}}, 0\right) \Psi_{\varepsilon \kappa m_{j}}(\mathbf{r}) .
$$

Here $\kappa=(-1)^{l+j+1 / 2}(j+1 / 2)$ is the Dirac quantum number determined by the angular momentum $j$ and the parity $l, C_{j_{1} m_{1} j_{2} m_{2}}^{J M}$ is the Clebsh-Gordan coefficient, $\delta_{j, l}$ is the phase shift induced by the scattering potential, $D_{M M^{\prime}}^{J}$ is the Wigner matrix [25, 26], angles $\varphi_{\hat{\nu}}$ and $\theta_{\hat{\nu}}$ describe the incident electron, and $\Psi_{\varepsilon \kappa m_{j}}(\mathbf{r})$ is the partial-wave solution of the Dirac equation in the scattering potential $V(r)$ whose explicit form will be specified below. Making use of Eqs. (4), (5), and (6) one can obtain the explicit expression for the bispinor amplitude $G_{\mu_{i}}^{(+)}(\hat{\boldsymbol{\nu}}, \hat{\mathbf{n}})$ :

$$
G_{\mu_{i}}^{(+)}(\hat{\boldsymbol{\nu}}, \hat{\mathbf{n}})=\frac{1}{\sqrt{2 \varepsilon(2 \pi)^{3}}}\left(\begin{array}{l}
\sqrt{\varepsilon+1} \\
\sqrt{\varepsilon-1}(\boldsymbol{\sigma} \cdot \hat{\mathbf{n}})
\end{array}\right)[A+2 B(\hat{\boldsymbol{\eta}} \cdot \mathbf{S})] \chi_{1 / 2 \mu_{i}}(\hat{\boldsymbol{\nu}}),
$$


where $\hat{\boldsymbol{\eta}}=[\hat{\boldsymbol{\nu}} \times \hat{\mathbf{n}}] /|[\hat{\boldsymbol{\nu}} \times \hat{\mathbf{n}}]|$ and the scattering amplitudes $A$ and $B$ express as $[21]$ :

$$
\begin{aligned}
& A \equiv A(\hat{\boldsymbol{\nu}} \cdot \hat{\mathbf{n}})=\frac{1}{2 i p} \sum_{l=0}^{\infty}\left\{(l+1)\left[\exp \left(2 i \delta_{l+1 / 2, l}\right)-1\right]+l\left[\exp \left(2 i \delta_{l-1 / 2, l}\right)-1\right]\right\} P_{l}(\hat{\boldsymbol{\nu}} \cdot \hat{\mathbf{n}}), \\
& B \equiv B(\hat{\boldsymbol{\nu}} \cdot \hat{\mathbf{n}})=\frac{1}{2 p} \sum_{l=1}^{\infty}\left[\exp \left(2 i \delta_{l+1 / 2, l}\right)-\exp \left(2 i \delta_{l-1 / 2, l}\right)\right] P_{l}^{1}(\hat{\boldsymbol{\nu}} \cdot \hat{\mathbf{n}}) .
\end{aligned}
$$

Here $P_{l}$ and $P_{l}^{1}$ are the Legendre polynomials and associated Legendre functions, respectively. Substituting Eqs. (3) and (17) into Eq. (2) one can express the amplitude of the process in terms of the amplitudes $A$ and $B$ :

$$
\tau_{\mu_{f} \mu_{i}}^{(\mathrm{pl})}=\chi_{1 / 2 \mu_{f}}^{\dagger}(\hat{\mathbf{n}})[A+2 B(\hat{\boldsymbol{\eta}} \cdot \mathbf{S})] \chi_{1 / 2 \mu_{i}}(\hat{\boldsymbol{\nu}})
$$

and, as a consequence, completely describe the process of the elastic electron scattering. As an example, the DCS for the unpolarized incident electron beam expresses as

$$
\frac{d \sigma^{(\mathrm{pl})}}{d \Omega}=\frac{1}{2} \sum_{\mu_{f} \mu_{i}}\left|\tau_{\mu_{f} \mu_{i}}^{(\mathrm{pl})}\right|^{2}=|A|^{2}+|B|^{2}
$$

\section{B. Mott scattering of the twisted electrons}

Above we have briefly recalled the theory of the elastic scattering of the conventional (planewave) electrons. Now we proceed to the consideration of the Mott scattering of the twisted electrons.

Let us start with the short theoretical description of twisted electrons. In the present study we restrict ourselves to the case of so-called Bessel electrons. Free twisted electrons in such a form are the solutions of the Dirac equation in an empty space with the well-defined energy $\varepsilon$, the helicity $\mu$, and the projections of the linear momentum $p_{z}$ and the total angular momentum $m$ onto the propagation direction. Here the $z$-axis is fixed along this direction. In addition, the absolute value of the transverse momentum $\varkappa=\left(\varepsilon^{2}-1-p_{z}^{2}\right)^{\frac{1}{2}}$ is well defined. The explicit form of the wave function of the Bessel twisted electron is given by [14]:

$$
\psi_{\varkappa p_{z} m \mu}(\mathbf{r})=\int \frac{e^{i m \varphi_{p}}}{2 \pi p_{\perp}} \delta\left(p_{\|}-p_{z}\right) \delta\left(p_{\perp}-\varkappa\right) i^{\mu-m} \psi_{\mathbf{p} \mu}(\mathbf{r}) d \mathbf{p},
$$

where $p_{\|}$and $p_{\perp}$ are the longitudinal and transversal components of the momentum $\mathbf{p}$, respectively, and $\psi_{\mathbf{p} \mu}$ is the wave function of the plane-wave electron (5). From the form of the integrand in Eq. (12) it is seen that the vortex electron can be viewed as a coherent superposition of the 
plane-wave electrons with the momenta $\mathbf{p}$ uniformly distributed over the surface of a cone with the fixed opening angle $\theta_{p}=\arctan \left(\varkappa / p_{z}\right)$.

Having presented the theoretical description of the free vortex electrons, we now turn to the Mott scattering of these electrons by a single atom. To begin with, we fix the geometry of the considered process as shown in Fig. 1]

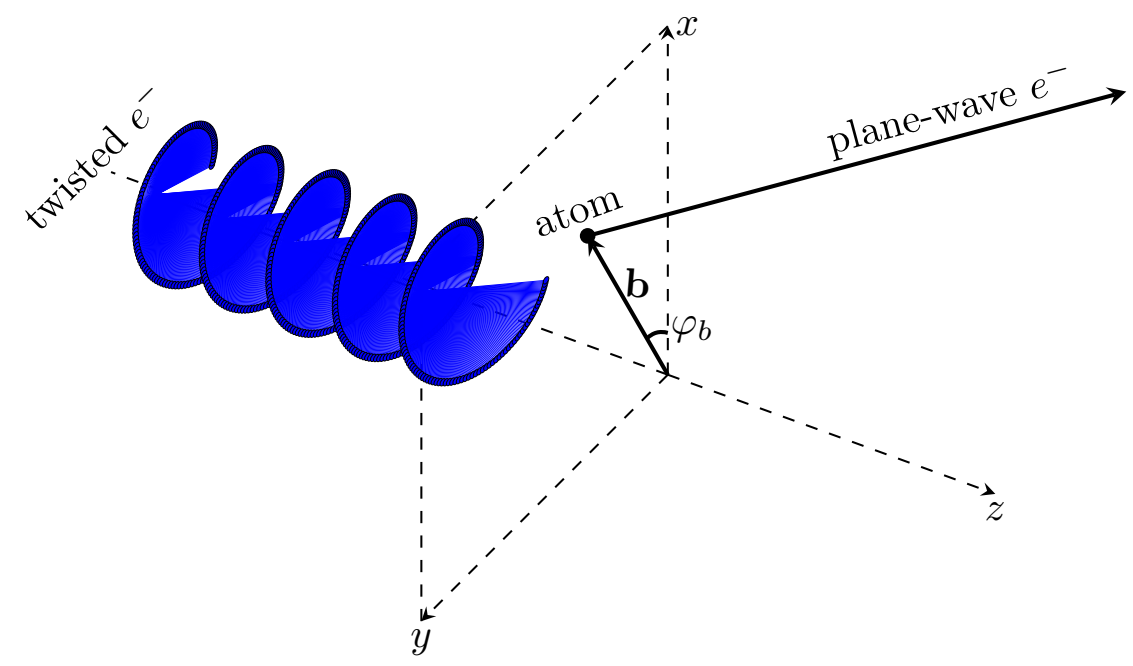

FIG. 1. The geometry of the Mott scattering of the twisted electron on the atomic target being shifted from the $z$ axis on the impact parameter $\mathbf{b}$.

In this figure the Mott scattering of the twisted electron by a single atom being shifted from the $z$ axis on the impact parameter $\mathbf{b}=\left(\mathrm{b}_{\mathrm{x}}, \mathrm{b}_{\mathrm{y}}, 0\right)$ is depicted. It should be noted that the relative position of the target and the incident vortex electron is important. This is explained by the fact that the intensity profile and the flow of the twisted electron are not homogeneous functions of the space variables [14, 15]. Additionally, here and throughout we consider that the scattered electron is asymptotically described as the plane-wave one. This is done in accordance with the assumption that in the experiments the detectors of the plane-wave electrons are utilized.

Let us now proceed to the theoretical description of the Mott scattering of the twisted electrons. This description can be naturally performed via the construction of the amplitude of the process considered. In order to construct the amplitude incorporating the electron-atom interaction nonperturbatively, one can utilize the wave function of the twisted electron being a solution of the Dirac equation in the external field of the corresponding atomic target. The explicit form 
of such a wave function for the process depicted in Fig. [1 is [15]

$$
\Psi_{\varkappa m p_{z} \mu_{i}}^{(+)}(\mathbf{r}+\mathbf{b})=\int \frac{e^{i m \varphi_{p}}}{2 \pi p_{\perp}} \delta\left(p_{z}-p_{\|}\right) \delta\left(p_{\perp}-\varkappa\right) i^{\mu_{i}-m} e^{i \mathbf{p} \cdot \mathbf{b}} \Psi_{\mathbf{p} \mu_{i}}^{(+)}(\mathbf{r}) d \mathbf{p} .
$$

The subsequent construction of the amplitude of the Mott scattering is performed in a way similar to one presented in Section IIA. In the framework of this approach, the amplitude for the scattering of Bessel electrons depicted in Fig. 1 can be written as

$$
\tau_{\mu_{f} \mu_{i}}^{(\mathrm{tw})}=\sqrt{(2 \pi)^{3}} U_{\mathbf{p}_{f} \mu_{f}}^{\dagger}(\hat{\mathbf{n}}) G_{m \mu_{i}, \mathbf{b}}^{(+)}\left(\theta_{p}, \hat{\mathbf{n}}\right)
$$

where $G_{m \mu_{i}, \mathbf{b}}^{(+)}\left(\theta_{p}, \hat{\mathbf{n}}\right)$ is the bispinor amplitude which is defined as follows

$$
\Psi_{\varkappa m p_{z} \mu_{i}}^{(+)}(\mathbf{r}+\mathbf{b}) \underset{r \rightarrow \infty}{\longrightarrow} \psi_{\varkappa m p_{z} \mu_{i}}(\mathbf{r}+\mathbf{b})+G_{m \mu_{i}, \mathbf{b}}^{(+)}\left(\theta_{p}, \hat{\mathbf{r}}\right) \frac{e^{i p r}}{r}
$$

From Eq. (15) one can derive

$$
G_{m \mu_{i}, \mathbf{b}}^{(+)}\left(\theta_{p}, \hat{\mathbf{r}}\right)=\int d \mathbf{p} \frac{e^{i m \varphi_{p}}}{2 \pi p_{\perp}} \delta\left(p_{\|}-p_{z}\right) \delta\left(p_{\perp}-\varkappa\right) i^{\mu_{i}-m} e^{i \mathbf{p} \cdot \mathbf{b}} G_{\mu_{i}}^{(+)}(\hat{\mathbf{p}}, \hat{\mathbf{r}}),
$$

where $\hat{\mathbf{p}}$ is the unit vector in the $\mathbf{p}$ direction and $G_{\mu_{i}}^{(+)}(\hat{\mathbf{p}}, \hat{\mathbf{r}})$ is the bispinor amplitude of the elastic scattering of the plane-wave electrons (7). Inserting Eq. (16) into Eq. (14), one obtains the amplitude for the Mott scattering of the twisted electron by the single atom

$$
\tau_{\mu_{f} \mu_{i}}^{(\mathrm{tw})}(\mathbf{b})=\int d \mathbf{p} \frac{e^{i m \varphi_{p}}}{2 \pi p_{\perp}} \delta\left(p_{z}-p_{\|}\right) \delta\left(p_{\perp}-\varkappa\right) i^{\mu_{i}-m} e^{i \mathbf{p} \cdot \mathbf{b}} \tau_{\mu_{f} \mu_{i}}^{(\mathrm{pl})} .
$$

Since this amplitude contains the complete information about the process under investigation we regard the theoretical description of the Mott scattering of the twisted electrons as complete.

Until now we have discussed the elastic scattering of the twisted electron by a single atom. This process is interesting from theoretical viewpoint but it can hardly be realized in experiment. We consider, therefore, a more realistic scenario in which the twisted electron beam collides with a macroscopic target, which we describe as an incoherent superposition of atoms being randomly and homogeneously distributed. The differential cross section for this case is given by [14]:

$$
\frac{d \sigma_{\mu_{f} \mu_{i}}^{(\mathrm{tw})}}{d \Omega}=\int \frac{d \mathbf{b}}{\pi R^{2}}\left|\tau_{\mu_{f} \mu_{i}}^{(\mathrm{tw})}(\mathbf{b})\right|^{2}=\frac{1}{\cos \theta_{p}} \int \frac{d \varphi_{p}}{2 \pi} \frac{d \sigma_{\mu_{f} \mu_{i}}^{(\mathrm{pl})}}{d \Omega}
$$

where $1 /\left(\pi R^{2}\right)$ is the cross section area with $R$ being the radius of the cylindrical box. From Eq. (18) it is seen that in the case of the elastic scattering of the twisted electrons by the macroscopic target the DCS does not depend on the TAM projection. 


\section{RESULTS AND DISCUSSIONS}

Here we restrict our consideration to the Mott scattering of the twisted electrons by the target consisting of the neutral atoms. Following work [14], we describe the electrostatic potential of a neutral atom as was suggested in Refs. [27, 28], namely, by a sum of three Yukawa terms

$$
V(r)=-\frac{\alpha Z}{r} \sum_{i=1}^{3} A_{i} e^{-\alpha_{i} r},
$$

where $A_{i}$ is the amplitude of the potential and $\alpha_{i}$ is the scaling constant. In the framework of the Born approximation, this potential allows one to obtain the analytical expression for the Mott scattering amplitude [14]. In order to obtain the results beyond the Born approximation, one needs to evaluate the amplitudes (8) and (9) which determine uniquely the amplitudes (14) and (17). This requires the knowledge of the phase shifts $\delta_{j, l}$ being induced by the scattering potential (19). Here these phases are numerically found with the usage of the modified RADIAL package [29]. As an independent check, the phase shifts $\delta_{j, l}$ were found by means of the variable phase method [30 32].

\section{A. Total scattering cross sections}

Let us compare the total cross sections obtained within the Born, $\sigma_{\text {tot }}^{(\mathrm{tw}, \mathrm{B})}$, and all-order, $\sigma_{\text {tot }}^{(\mathrm{tw})}$, approaches. For this purpose, we introduce the ratio of the cross sections:

$$
\mathscr{R}=\frac{\sigma_{\text {tot }}^{(\mathrm{tw}, \mathrm{B})}}{\sigma_{\mathrm{tot}}^{(\mathrm{tw})}} .
$$

The explicit expression for $\sigma_{\text {tot }}^{(\mathrm{tw}, \mathrm{B})}$ can be found in Ref. [14]. The all-order relativistic total cross section, $\sigma_{\text {tot }}^{(\mathrm{tw})}$, is obtained from Eq. (18) via integration over the solid angle of the scattered electron and performing the summation and averaging over the final- and initial-state helicities, respectively. Here it is worth stressing that the $\mathscr{R}$ parameter does not depend on the opening (conical) angle of the vortex electron $\theta_{p}$. Indeed, from Eq. (18) one can deduce that the total cross sections for the Mott scattering of the twisted and plane-wave electrons are connected as follows

$$
\sigma_{\mathrm{tot}}^{(\mathrm{tw})}=\frac{\sigma_{\mathrm{tot}}^{(\mathrm{pl})}}{\cos \theta_{p}}
$$


From this equation one can conclude that the $\mathscr{R}$ parameter coincides with the same parameter for the plane-wave electrons.

In Fig. 2 the ratio (201) is presented as a function of the incident electron energy. It should be noted that this ratio can be also obtained from the results of Ref. [27] where the Mott scattering of the plane-wave electrons has been studied. The values presented in Fig. 2 are in excellent agreement with ones from that paper, what supports our numerical calculations.

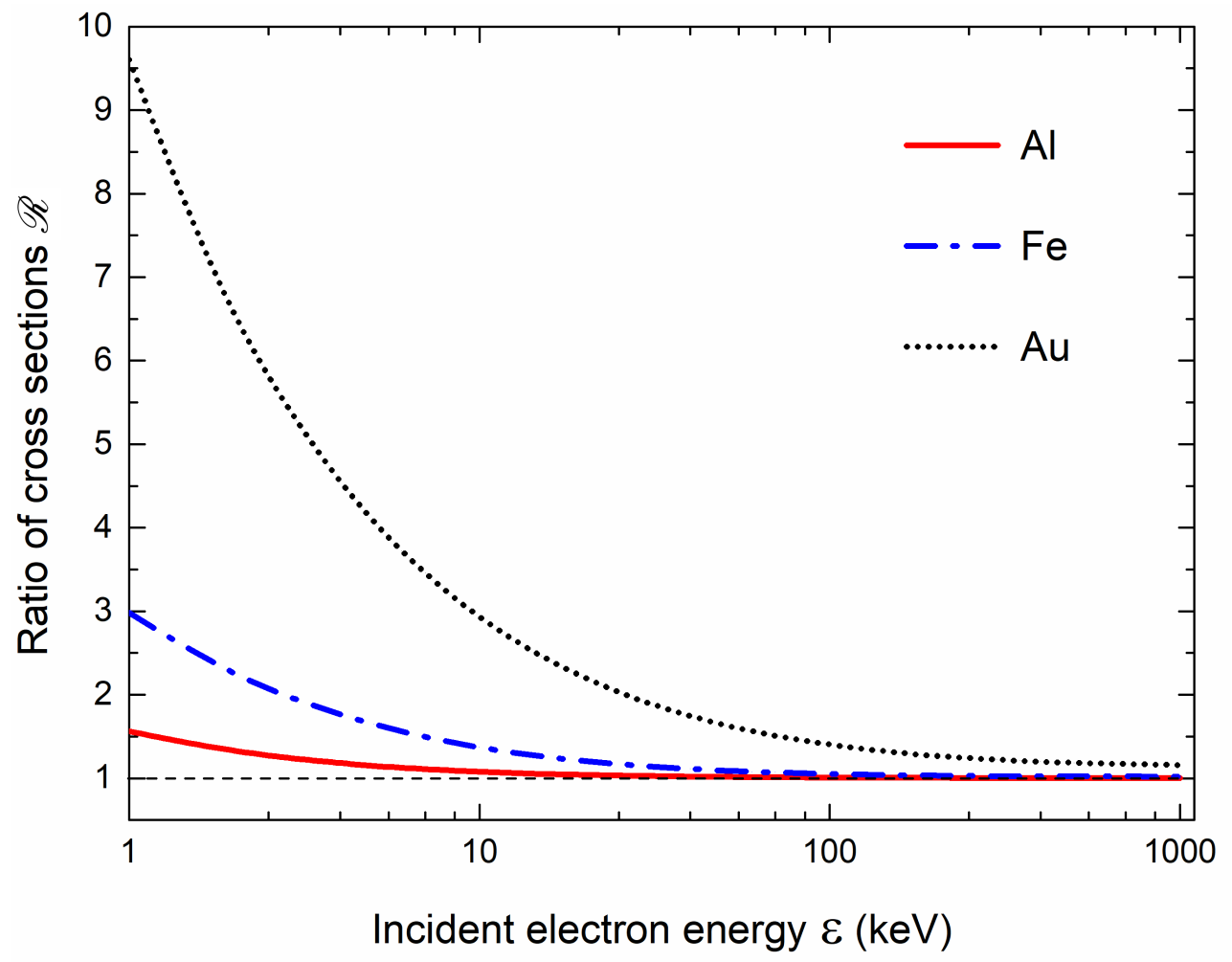

FIG. 2. The $\mathscr{R}$ parameter (20) as a function of the incident electron energy. The solid (red), dashdotted (blue), and dotted (black) lines correspond to the scattering by the aluminium $(Z=13)$, iron $(Z=26)$, and gold $(Z=79)$ mactroscopic targets, respectively.

From this figure one can see that for the scattering by such light atoms as aluminium $(Z=13)$ the results obtained within the Born approximation agree with the all-order relativistic ones on the level better than $10 \%$ already at energies higher than $10 \mathrm{keV}$. In the case of the elastic scattering by iron $(Z=26)$ atoms which was studied in Ref. [14] this accuracy can be achieved only at energies higher than $100 \mathrm{keV}$ and amounts to few percents for $1 \mathrm{MeV}$. This means that the reliable value of the total cross section for the Mott scattering of the twisted electrons 
with energies around (and lower) $100 \mathrm{keV}$ can be obtained only within the all-order relativistic treatment. For the Mott scattering by the gold $(Z=79)$ target, meanwhile, the difference between the results obtained in the Born approximation and beyond is larger than $15 \%$ at 1 $\mathrm{MeV}$ and reaches $40 \%$ at energies around $100 \mathrm{keV}$. One can conclude, therefore, that the Born approximation can not be applied for such heavy systems as gold $(Z=79)$ for energies up to 1 $\mathrm{MeV}$.

\section{B. Differential cross sections and degree of polarization}

Let us now compare the differential cross sections calculated within the Born and all-order relativistic approach. To this end, in Fig. 3 we present the DCS for the Mott scattering of the twisted electron with the opening (conical) angle $\theta_{p}=40^{\circ}$ by the macroscopic iron $(Z=26)$ target.

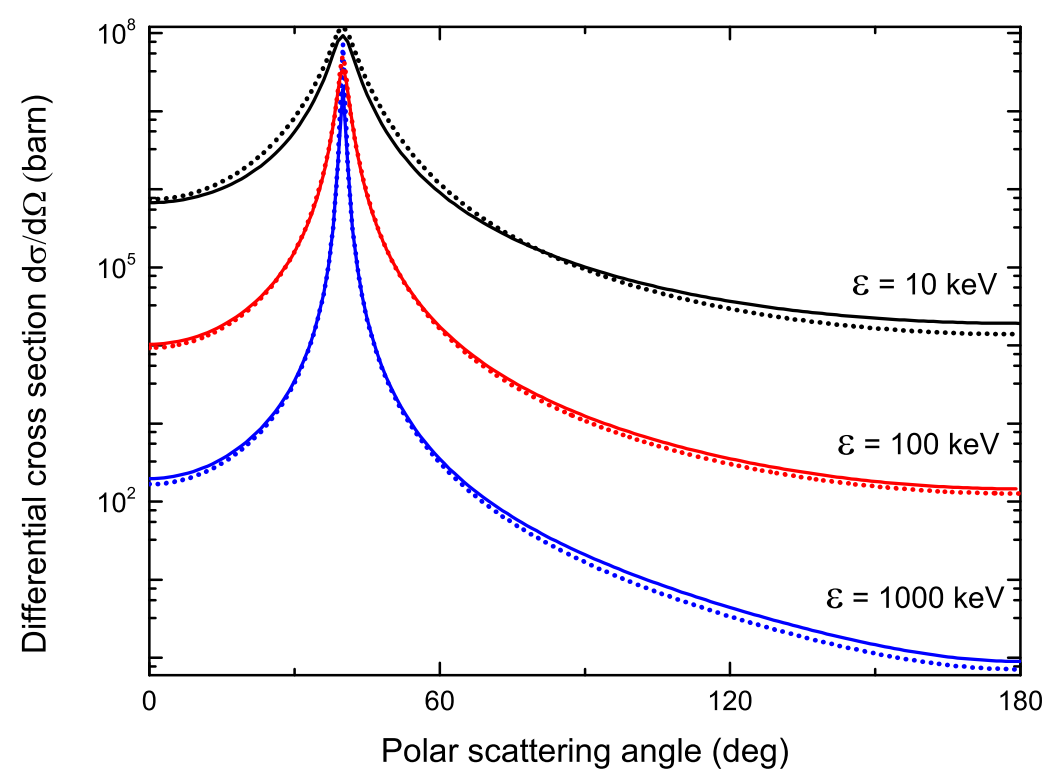

FIG. 3. The differential cross section for the Mott scattering of the twisted electrons with the opening (conical) angle $\theta_{p}=40^{\circ}$ by the macroscopic iron $(Z=26)$ target. Results of the Born approximation (dotted lines) are compared with predictions of the all-order relativistic approach (solid lines). The kinetic energies of the incident electron are $10 \mathrm{keV}, 100 \mathrm{keV}$, and $1 \mathrm{MeV}$. 
From this figure it is seen that the qualitative behavior of the DCS can be well described in the framework of the Born approximation [14]. Indeed, as in the Born approximation, the DCS for the Mott scattering of the twisted electrons has a peak at polar scattering angle $\theta=\theta_{p}$. However, it is worth stressing that the absolute value of the DCS obtained in this approximation can strongly differ from the all-order relativistic result. For instance, in the case of the Mott scattering of the $100 \mathrm{keV}$ twisted electron the difference between the DCS obtained within the Born approximation and the all-order approach reaches $16 \%$ for the scattering angle $120^{\circ}$.

So far we have compared the total and differential cross sections obtained within the Born approximation and beyond it. For both these measurables the necessity of the all-order relativistic calculations was found. From our point of view, it is also important to investigate the effects beyond the Born approximation for the relative measurable quantities. In the framework of the present investigation, we consider one of them, namely, the degree of longitudinal polarization of the scattered electrons $P$ :

$$
P=\frac{d \sigma_{1 / 2,1 / 2}-d \sigma_{1 / 2,-1 / 2}}{d \sigma_{1 / 2,1 / 2}+d \sigma_{1 / 2,-1 / 2}},
$$

where $d \sigma_{\mu_{f} \mu_{i}} \equiv \frac{d \sigma_{\mu_{f} \mu_{i}}}{d \Omega}$ and it is assumed that the incident electron is completely longitudinally polarized $\left(\mu_{i}=1 / 2\right)$. In Fig. 4 we present the degree of the longitudinal polarization $P$ for the Mott scattering of the twisted electron by the macroscopic iron $(Z=26)$ target.
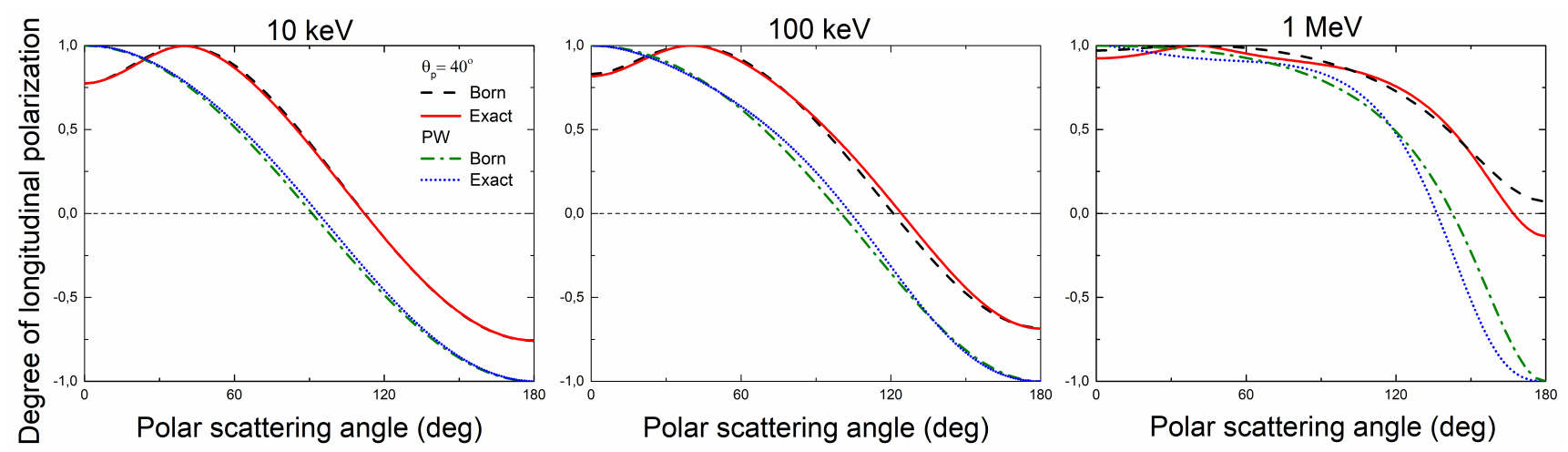

FIG. 4. The degree of the longitudinal polarization $P$, defined by Eq. (22), for the Mott scattering of the twisted and plane-wave electrons by the macroscopic iron $(Z=26)$ target. Calculations within the all-order approach and Born approximation have been performed for the kinetic energy $\varepsilon=10 \mathrm{keV}$ (left panel), $100 \mathrm{keV}$ (middle panel) and $1 \mathrm{MeV}$ (right panel).

From this figure one can see that for the kinetic energies of the incident electron $10 \mathrm{keV}$ and 100 
$\mathrm{keV}$ (left and middle panels, respectively) the degree of polarization obtained within the Born approximation almost coincide with the all-order result. The most interesting situation occurs for the Mott scattering of the $1 \mathrm{MeV}$ electron (right panel in Fig. 4). For this energy one might expect a better agreement of the results obtained within the Born approximation and the allorder relativistic treatment. However, our calculations show that the corresponding difference of the degree of polarization is the most pronounced for the Mott scattering of the $1 \mathrm{MeV}$ electron. From our point of view, it can be explained by the enhancement of the spin effects with the increase of the incident electron energy which are not accounted in the framework of the Born approximation [21].

\section{Effects of kinematic properties of twisted electrons}

We finish this section by investigating the dependence of the angular and polarization properties of the Mott scattering on the kinematic parameters of the incident twisted electron. In particular, the sensitivity of the differential cross section and the degree of the longitudinal polarization to variations of the energy and the opening (conical) angle of the vortex electron is studied. In the present section, we investigate the dependence from the kinematic properties of the incident twisted electrons for such a heavy element as gold $(Z=79)$. Additional interest to this system is explained by the fact that the golden foil is usually used in Mott detectors [33, 34]. As shown in Section $\amalg$, the description of this process can not be performed within the Born approximation. Below we, therefore, present only the results of the all-order calculations.

The DCS for the Mott scattering by the macroscopic gold target is presented in Fig. 5. From this figure it is seen that the DCS for the Mott scattering by the golden foil does not differ qualitatively from the DCS for the elastic scattering by other macroscopic targets (see Section IIIA and Ref. [14]). Namely, the electrons are predominantly scattered under the angle $\theta=\theta_{p}$. And, although the qualitative behavior of the DCS for the scattering by the macroscopic gold target can be well described in the framework of the Born approximation, we would like to stress that the results obtained within this approximation can not be regarded as reliable. The degree of the polarization $P$ for the Mott scattering by the macroscopic gold target is depicted in Fig. 6. This figure demonstrates the strong dependence of $P$ from the opening angle $\theta_{p}$ for all energies of the incident electron. Moreover, this dependence becomes the most prominent for large energies 


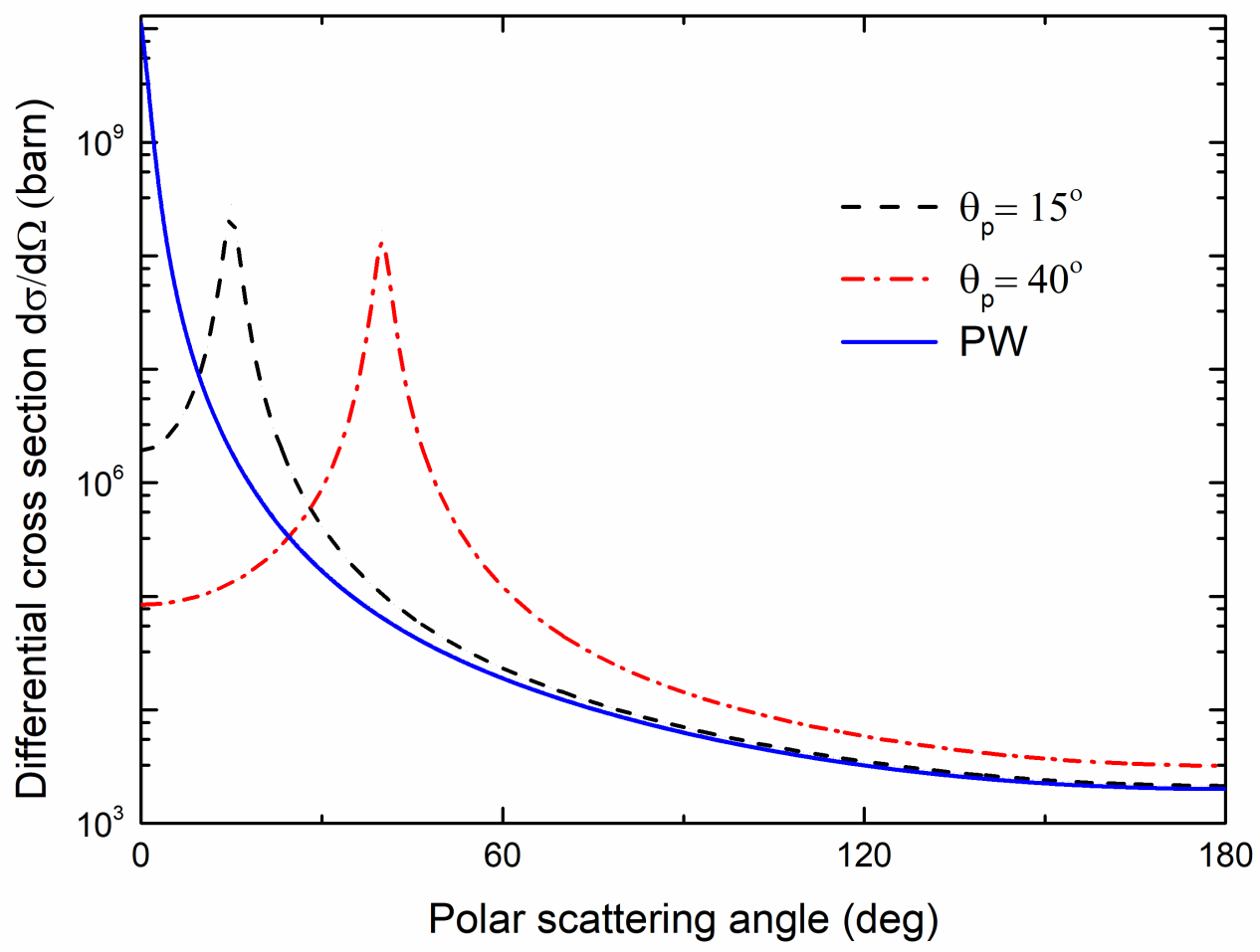

FIG. 5. The differential cross sections for the Mott scattering of $100 \mathrm{keV}$ electrons by the macroscopic gold $(Z=79)$ target. The solid (blue) line corresponds to the plane-wave case, meanwhile the dashed (black) and dash-dotted (red) lines are related to the scattering of the twisted electron with the opening angles $\theta_{p}=15^{\circ}$ and $40^{\circ}$, respectively.

(see the right panel in Fig. 6). From this panel it is also seen that at certain conical angles the degree of the longitudinal polarization is strictly positive.

\section{CONCLUSION}

In the present work, we have considered the Mott scattering of the twisted electrons by the atomic targets taking into account the electron-atom interaction in all orders. For the nonperturbative treatment of this interaction the method based on the results of Ref. [15] was employed. In the framework of this method, the differential and total cross sections and the degree of longitudinal polarization for the Mott scattering of the twisted electrons by various macroscopic targets were evaluated.

The comparison of the results being nonperturbative in electron-atom interaction with ones 

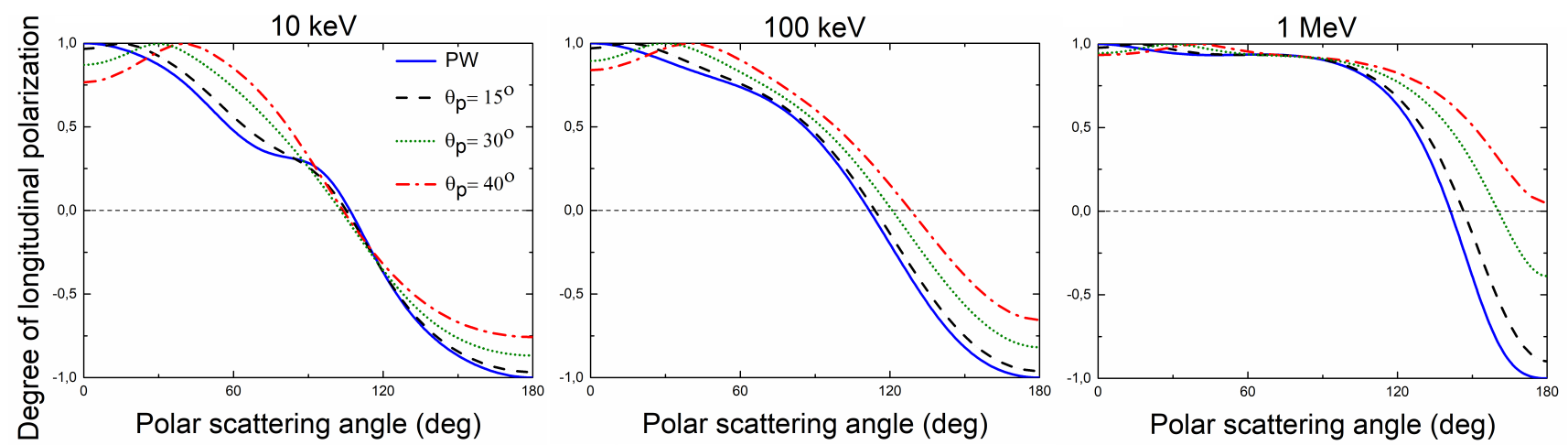

FIG. 6. The degree of polarization $P$, defined by Eq. (22), for the Mott scattering by the macroscopic gold $(Z=79)$ target. The solid (blue) line corresponds to the plane-wave case, meanwhile the dashed (black), dotted (green) and dash-dotted (red) lines are related to the scattering of the twisted electron with the opening angles $\theta_{p}=15^{\circ}, \theta_{p}=30^{\circ}$, and $\theta_{p}=40^{\circ}$, respectively. The left, middle, and right panels correspond to the cases of $10 \mathrm{keV}, 100 \mathrm{keV}$, and $1 \mathrm{MeV}$ kinetic energies of the incident electron, respectively.

obtained within the Born approximation was performed. It was found that the reliable value of the total cross section for the Mott scattering of the twisted electrons with energies around (and lower) $100 \mathrm{keV}$ by the iron $(Z=26)$ target can be obtained only within the all-order relativistic treatment. In the case of the scattering by the gold $(Z=79)$ target it was found that the Born approximation can not be applied for energies lower than $1 \mathrm{MeV}$. In contrast, the qualitative behaviour of the differential cross section and the degree of longitudinal polarization can be well described in this approximation.

Additionally, the results obtained within the all-order relativistic treatment were presented for the elastic scattering of the twisted electrons at the golden foil of the Mott detector. For such target the strong dependence of the degree of the longitudinal polarization from the opening angle $\theta_{p}$ was found.

\section{ACKNOWLEDGEMENTS}

This work was supported by the grant of the President of the Russian Federation (Grant No. MK-4468.2018.2), by RFBR (Grants No. 18-32-00602 and No. 16-02-00334), by the German- 
Russian Interdisciplinary Science Center (G-RISC) funded by the German Federal Foreign Office via the German Academic Exchange Service (DAAD), and by SPbSU-DFG (Grants No. 11.65.41.2017 and No. STO 346/5-1).

[1] K. Y. Bliokh, Y. P. Bliokh, S. Savel'ev, and F. Nori, Phys. Rev. Lett. 99, 190404 (2007).

[2] J. Verbeeck, H. Tian, and P. Schattschneider, Nature 467, 301 (2010).

[3] M. Uchida and A. Tonomura, Nature 464, 737 (2010).

[4] B. J. McMorran, A. Agrawal, I. M. Anderson, A. A. Herzing, H. J. Lezec, J. J. McClelland, and J. Unguris, Science 331, 192 (2011).

[5] K. Y. Bliokh et al., Phys. Rep. 690, 1 (2017).

[6] E. Mafakheri, A. H. Tavabi, P.-H. Lu, R. Balboni, F. Venturi, C. Menozzi, G. C. Gazzadi, S. Frabboni, A. Sit, R. E. Dunin-Borkowski, E. Karimi, and V. Grillo, Appl. Phys. Lett. 110, 093113 (2017).

[7] J. Rusz and S. Bhowmick, Phys. Rev. Lett. 111, 105504 (2013).

[8] A. Béché, R. Van Boxem, G. Van Tendeloo, and J. Verbeeck, Nat. Phys. 10, 26 (2014).

[9] P. Schattschneider, S. Löffler, M. Stöger-Pollach, and J. Verbeeck, Ultramicroscopy 136, 81 (2014).

[10] A. Edström, A. Lubk, and J. Rusz, Phys. Rev. Lett. 116, 127203 (2016).

[11] R. V. Boxem, B. Partoens, and J. Verbeeck, Phys. Rev. A 89, 032715 (2014).

[12] O. Matula, A. G. Hayrapetyan, V. G. Serbo, A. Surzhykov, and S. Fritzsche, New J. Phys. 16, 053024 (2014).

[13] R. V. Boxem, B. Partoens, and J. Verbeeck, Phys. Rev. A 91, 032703 (2015).

[14] V. G. Serbo, I. P. Ivanov, S. Fritzsche, D. Seipt, and A. Surzhykov, Phys. Rev. A 92, 012705 (2015).

[15] V. A. Zaytsev, V. G. Serbo, and V. M. Shabaev, Phys. Rev. A 95, 012702 (2017).

[16] T. Schachinger, S. Löffler, A. Steiger-Thirsfeld, M. Stöger-Pollach, S. Schneider, D. Pohl, B. Rellinghaus, and P. Schattschneider, Ultramicroscopy 179, 15 (2017).

[17] D. S. Negi, J. C. Idrobo, and J. Rusz, Sci. Rep. 8, 4019 (2018)

[18] D. Dutta, talk given at 22nd International Spin Symposium, Urbana-Champaign, USA, 2016.

[19] N. F. Mott and H. S. W. Massey, The Theory of Atomic Collisions (Clarendon Press, Oxford, 1965). 
[20] A. I. Akhiezer and V. B. Berestetskii, Quantum Electrodynamics (Interscience, New York, 1965).

[21] V. B. Berestetsky, E. M. Lifshitz, and L. P. Pitaevskii, Quantum Electrodynamics (ButterworthHeinemann, Oxford, 2006).

[22] M. E. Rose, Relativistic Electron Theory, (Wiley, New York, 1961).

[23] R. H. Pratt, A. Ron, and H. K. Tseng, Rev. Mod. Phys. 45, 273 (1973); 45, 663(E) (1973).

[24] J. Eichler and W. Meyerhof, Relativistic Atomic Collisions, (Academic, San Diego, 1995).

[25] M. E. Rose, Elementary Theory of Angular Momentum, (Wiley, New York, 1957).

[26] D. A. Varshalovich, A. N. Moskalev, and V. K. Khersonskii, Quantum Theory of Angular Momentum, (World Scientific, Singapore, 1988).

[27] F. Salvat, Phys. Rev. A 43, 578 (1991).

[28] F. Salvat, J. D. Martinez, R. Mayol, and J. Parellada, Phys. Rev. A 36, 467 (1987).

[29] F. Salvat, J. M. Fernandez-Varea, W. Williamson Jr., Comput. Phys. Commun. 90, 151 (1995).

[30] F. Calogero, Variable Phase Approach to Potential Scattering, (Academic, New York, 1967).

[31] V. V. Babikov, Phase Function Method in Quantum Mechanics, (Nauka, Moscow, 1968).

[32] D. P. Grechukhin and A. V. Lomonosov, JETP Lett. 60, 779 (1994).

[33] N. F. Mott, Proc. R. Soc. (London) 135, 429 (1932).

[34] J. Kessler, Polarized electrons (Springer, Berlin, 1985). 